\title{
Depression and its associated factors among HIV/AIDS patients attending ART clinics at Gimbi General hospital, West Ethiopia, 2018
}

\author{
Muktar Abadiga*
}

\begin{abstract}
Objective: The aim of this study was to assess the prevalence and factors associated with depression among people living with HIV/AIDS attending Gimbi General hospital, West Ethiopia. Institutional based cross-sectional study was conducted on 404 HIV/AIDS patients, from March 01 to March 30, 2018. Multivariable logistic regression was used to determine factors associated with depression. Possible association and statistical significance were measured using odds ratio at 95\% confidence interval and P-value less than 0.05 .

Results: A total of 393 HIV/AIDS patients were included in this study. Out of this, $41.7 \%$ had depression. Perceived social stigma ( $A O R=6.98,95 \% \mathrm{Cl} 3.07,15.86)$, opportunistic infection ( $\mathrm{OOR}=9.38,95 \% \mathrm{Cl} 4.21,20.89$ ), adverse drug reaction $(A O R=3.73,95 \% \mathrm{Cl} 1.58,8.81)$, absence of family/social support ( $\mathrm{AOR}=9.97,95 \% \mathrm{Cl} 3.57,27.86)$, and presence of other chronic diseases (AOR $=6.14,95 \% \mathrm{Cl} 1.66,22.68$ ) were significantly associated with depression. The level of depression among HIV/AIDS patient in this study was high. The clinician should early recognize and treat drug side effects, early detect and manage opportunistic infection and other chronic diseases, and give health information about the disease for the community to reduce social stigma.
\end{abstract}

Keywords: Depression, HIV/AIDS patients, Gimbi General hospital

\section{Introduction}

Depression is a common mental disorder presents with depressed mood, loss of interest, feelings of guilt, disturbed sleep, and poor concentration [1]. It is the leading cause of disability and the fourth leading contributor to the global burden of disease [2, 3]. About 350 million people were affected worldwide, and the life-time risk of depression is one in five women and one in ten men $[4,5]$. In Ethiopia, the pooled prevalence of depression is $11 \%$ [6]. Depression is one of the most common mental disorders people with HIV/AIDS experience [7, 8]. The prevalence of depression on HIV-infected patients in Brazil is $32 \%$ [9]. Other studies conducted in different countries on prevalence of depression among HIV patients showed 26.7\% in Cameroon [10], 18.9\% in Malawi [11] and 40.9\% in China [12]. In Ethiopia, the study done in different

\footnotetext{
*Correspondence: muktarabadiga@gmail.com
}

School of Nursing and Midwifery, Institute of Health Sciences, Wollega

University, Nekemte, Ethiopia part of a country showed $48.6 \%$ in Hawassa [13], $14.6 \%$ in Aksum town [14], 45.8\% in Harar town [15], and 35.5\% in Addis Ababa [16]. The level of depression is related to the severity of symptoms of HIV infection, and the presence of HIV/AIDS increases the risk of mental disorders [17-19].

Depression and HIV/AIDS are estimated to be the world's two leading causes of disability by 2030 [20]. Depression among PLHIV influences not only the health status of PLHIV but also has a negative effect on ART adherence [21]. Depression affects the ability to comply with HIV/AIDS treatment, as well as quality of life and lifespan [22, 23]. Depression also has destructive effects on the self-care behaviors necessary for management of HIV [24]. Studies showed that factors such as unemployment, age, CD4 count, sex, educational status, drug side effects, family/social support, stigma, stage of HIV, living companion, marital status, monthly income and opportunistic infection were predisposed HIV/AIDS patients to depression $[10-16]$. 
Although the prevalence of depression is high among HIV/AIDS patients, few studies have been conducted in Ethiopia and no study has been conducted at Gimbi General hospital. This study was done to fill this research gap which might provide evidence for the future's effective prevention and treatment of depression in people living with HIV/AIDS. Therefore; this study was aimed to assess the prevalence and associated factors of depression among people living with HIV/AIDS attending Gimbi General hospital, West Ethiopia.

\section{Main text Methods \\ Study setting and population}

This study was conducted at Gimbi General hospital from March 01 to March 30, 2018. The institutional based cross-sectional study design was employed. All ART patients on treatment follow up at Gimbi General hospital was the source population and the sampled ART patients who had treatment follow up during the study period was the study population. Patients whose ages are 18 years and above were included in the study. Patients who are eligible but not willing to take part in the study were excluded.

\section{Sample size determination and sampling techniques}

The sample size was calculated using the formula for estimation of a single population proportion. A $48.6 \%$ (0.486) proportion of HIV/AIDS patients who had depression was taken from similar a study done in Hawassa University Comprehensive Specialized Hospital [13], and by adding a non-response rate of $5 \%$, a total of 404 ART patients were enrolled in the study. Simple random sampling method was used to select the study participants.

\section{Data collection tool and procedures}

The data collection tool includes socio-demographic characteristics, clinical and behavioral variables, nineitem Patient Health Questionnaire (PHQ-9), The EightItem Morisky Medication Adherence Scale, Oslo 3-item social support scale and an 11-item HIV stigma scale. Depression was defined by a PHQ-9 score $\geq 5$ [25]. Adherence was defined as adherent with a Morisky Medication Adherence Scale score of 0 and non-adherent with a score of $\geq 1$. Oslo 3 -item social support scale has three categories: "poor support" 3-8, "moderate support" 9-11 and "strong support" 12-14 [26]. Study participants were classified as having or not having stigma using the mean of the stigma variable as cutoff point $[27,28]$.

\section{Data processing and analysis}

The data were coded, checked, cleaned and entered into Epi data version 3.1 and then exported into SPSS window version 20.0 for analysis. Bivariate analysis was done to find association between each independent variable with depression. Finally, multivariable logistic regression was used to find out the independent variables which best predict depression. Possible association and statistical significance were measured using odds ratio at $95 \%$ confidence interval and P-value less than 0.05.

\section{Data quality control}

The questionnaire was translated into the local language and then translated back to English by expertise for consistency. Five percent (5\%) of the questionnaire was pre-tested on ART patients having treatment follow up at Nekemte referral hospital. One-day training was also given for data collectors and supervisors.

\section{Results}

Socio-demographic characteristics of participants

Out of the total of 404 study participants sampled, 393 were participated; making a response rate of $97.3 \%$. From the total of 393 participants, 177 (45\%) were male, and 216 (55\%) were females. One hundred forty-two, (36.1\%) lie in the age group between 18 and 28 years, and the mean age was 25.6 years with \pm 9.45 SD. Regarding religion, $182(46.3 \%)$ were Protestants. Concerning marital status, 174 (44.3\%) were single followed by married, 132 (33.6\%). Concerning education, $168(42.7 \%)$ were completed grade 9-12, and 128 (32.6\%) were completed grade $1-8$. Regarding income, $108(27.5 \%)$ gets monthly income $<500$ Ethiopian birrs followed by $500-1000$ Ethiopian birr which accounts 98 (24.9\%) (Table 1).

\section{Clinical and behavioral characteristics of participants}

One hundred eighty-one (46.1\%) had CD4 count 200500 cells and 133 (33.8\%) had CD4 count 501-800 cells. Regarding the stage of HIV, 153 (38.9\%) were on stage II and $117(29.8 \%)$ were on stage I. One hundred seventyseven (45\%) had strong social support, 147 (37.4\%) had moderate and the remaining $69(17.6 \%)$ had poor social support. Majority of the study participants, 247 (62.8\%) don't experienced social stigma and 146 (37.2\%) had experienced social stigma. Majority of the study participants, $363(92.4 \%)$ had no history of other chronic illness and $270(68.7 \%)$ had no current opportunistic infections. Majority of the study participants, 268 (68.2\%) had no adverse drug reaction, and the remaining $125(31.8 \%)$ had experienced an adverse drug reaction (Table 2).

\section{Level of depression among the study participants}

The level of depression among the study participants were measured using nine-item Patient Health Questionnaire (PHQ-9) which is a validated depression screening tool having score range from 0 to 27. Study participants 
Table 1 Distribution of study participants by sociodemographic characteristics among HIV/AIDS patients at Gimbi General hospital, West Ethiopia, $2018(n=393)$

\begin{tabular}{|c|c|c|}
\hline Variables & Frequency & Percentage \\
\hline \multicolumn{3}{|l|}{ Sex } \\
\hline Male & 177 & 45 \\
\hline Female & 216 & 55 \\
\hline Total & 393 & 100 \\
\hline \multicolumn{3}{|l|}{ Ethnicity } \\
\hline Oromo & 234 & 59.5 \\
\hline Amhara & 106 & 27.0 \\
\hline Tigre & 24 & 6.1 \\
\hline Gurage & 16 & 4.1 \\
\hline Others & 13 & 3.3 \\
\hline Total & 393 & 100 \\
\hline \multicolumn{3}{|l|}{ Age } \\
\hline $18-28$ & 142 & 36.1 \\
\hline $29-38$ & 140 & 35.6 \\
\hline $39-48$ & 56 & 14.2 \\
\hline$\geq 48$ & 55 & 14.0 \\
\hline Total & 393 & 100 \\
\hline \multicolumn{3}{|l|}{ Religion } \\
\hline Protestant & 182 & 46.3 \\
\hline Orthodox & 106 & 27.0 \\
\hline Muslim & 49 & 12.5 \\
\hline Catholic & 46 & 11.7 \\
\hline Others & 10 & 2.5 \\
\hline Total & 393 & 100 \\
\hline \multicolumn{3}{|l|}{ Marital status } \\
\hline Married & 132 & 33.6 \\
\hline Single & 174 & 44.3 \\
\hline Divorced & 49 & 12.5 \\
\hline Widowed & 38 & 9.7 \\
\hline Total & 393 & 100 \\
\hline \multicolumn{3}{|l|}{ Educational status } \\
\hline No formal education & 30 & 7.6 \\
\hline Primary school (1-8) & 128 & 32.6 \\
\hline Secondary (9-10) & 168 & 42.7 \\
\hline Degree and above & 67 & 17.0 \\
\hline Total & 393 & 100 \\
\hline \multicolumn{3}{|l|}{ Occupational status } \\
\hline Gov'nt employee & 52 & 13.2 \\
\hline Private employee & 121 & 30.8 \\
\hline Farmer & 95 & 24.2 \\
\hline Merchant & 109 & 27.7 \\
\hline Others & 16 & 4.1 \\
\hline Total & 393 & 100 \\
\hline \multicolumn{3}{|l|}{ Monthly income } \\
\hline$<500$ & 108 & 27.5 \\
\hline $500-1000$ & 98 & 24.9 \\
\hline $1001-1500$ & 78 & 19.8 \\
\hline $1501-2000$ & 75 & 19.1 \\
\hline
\end{tabular}

Table 1 (continued)

\begin{tabular}{lcl}
\hline Variables & Frequency & Percentage \\
\hline$>200$ & 34 & 8.7 \\
Total & 393 & 100 \\
Living companion & & \\
Yes & 264 & 67.2 \\
No & 129 & 32.8 \\
Total & 393 & 100 \\
\hline
\end{tabular}

who scored PHQ-9 score $\geq 5$ were considered depressed and those who scored $<5$ were considered not depressed. Accordingly, out of the total of 393 study participants, 229 (58.3\%) had no depression and $164(41.7 \%)$ had depression.

\section{Bivariate logistic regression analysis}

In bivariate analysis, socio-demographic characteristics such as monthly income, occupation and living companion showed significant association with depression at P-value less than 0.25. Clinical variables such as CD4 cell count, HIV stage, adherence to medication, adverse drug reaction, history of other chronic illness and opportunistic infection showed significant association. Behavioral variables such as social support, substance use and perceived social stigma also showed significant association with depression at P-value less than 0.25 .

\section{Multivariate logistic regression analysis}

In the final model of logistic regression, social support, perceived social stigma, opportunistic infection, adverse drug reaction and presence of other chronic diseases were significantly associated with depression. Study participants who had poor social support were 9.97 times more likely to have depression than those who had strong social support (AOR $=9.97,95 \%$ CI 3.57, 27.86). Study participants who had experienced social stigma were 6.98 times more likely to develop depression than those who had no experienced social stigma $(\mathrm{AOR}=6.98,95 \% \mathrm{CI}$ $3.07,15.86)$. Respondents who developed adverse drug reactions were 3.73 times more likely to have depression than those who don't develop any adverse drug reaction $(\mathrm{AOR}=3.73,95 \%$ CI $1.58,8.81)$. Study participants who had history of other chronic diseases were 6.14 more likely to develop depression than those who had no other chronic illness $(A O R=6.14,95 \%$ CI 1.66, 22.68). Study participants who had history of opportunistic infection were 9.38 times more likely to have depression than those who had no history of opportunistic infection $(\mathrm{AOR}=9.38,95 \%$ CI 4.21, 20.89) (Table 3). 
Table 2 Distribution of study participants by clinical and behavioral factors among HIV/AIDS patients at Gimbi General hospital, West Ethiopia, $2018(n=393)$

\begin{tabular}{|c|c|c|}
\hline Variables & Frequency & Percentage \\
\hline \multicolumn{3}{|c|}{ CD4 cell count } \\
\hline$<200$ & 63 & 16.0 \\
\hline $200-500$ & 181 & 46.1 \\
\hline $501-800$ & 133 & 33.8 \\
\hline$>800$ & 16 & 4.1 \\
\hline Total & 393 & 100 \\
\hline \multicolumn{3}{|l|}{ Stage of HIV } \\
\hline Stage I & 117 & 29.8 \\
\hline Stage II & 153 & 38.9 \\
\hline Stage III & 72 & 18.3 \\
\hline Stage IV & 51 & 13.0 \\
\hline Total & 393 & 100 \\
\hline \multicolumn{3}{|c|}{ Social support } \\
\hline Poor & 69 & 17.6 \\
\hline Moderate & 147 & 37.4 \\
\hline Strong & 177 & 45.0 \\
\hline Total & 393 & 100 \\
\hline \multicolumn{3}{|c|}{ Perceived social stigma } \\
\hline Yes & 146 & 37.2 \\
\hline No & 247 & 62.8 \\
\hline Total & 393 & 100 \\
\hline \multicolumn{3}{|c|}{ Current substance use } \\
\hline Yes & 157 & 39.9 \\
\hline No & 236 & 60.1 \\
\hline Total & 393 & 100 \\
\hline \multicolumn{3}{|c|}{ Duration of disease } \\
\hline$<1$ year & 54 & 13.7 \\
\hline $1-5$ years & 137 & 34.9 \\
\hline $5-10$ years & 148 & 37.7 \\
\hline$>10$ years & 54 & 13.7 \\
\hline Total & 393 & 100 \\
\hline \multicolumn{3}{|c|}{ Adverse drug reaction } \\
\hline Yes & 125 & 31.8 \\
\hline No & 268 & 68.2 \\
\hline Total & 393 & 100 \\
\hline \multicolumn{3}{|c|}{ History of other chronic illness } \\
\hline Yes & 30 & 7.6 \\
\hline No & 363 & 92.4 \\
\hline Total & 393 & 100 \\
\hline \multicolumn{3}{|c|}{ History of Ols } \\
\hline Yes & 123 & 31.3 \\
\hline No & 270 & 68.7 \\
\hline Total & 393 & 100 \\
\hline \multicolumn{3}{|c|}{ Adherence to medication } \\
\hline Yes & 245 & 62.3 \\
\hline No & 148 & 37.7 \\
\hline Total & 393 & 100 \\
\hline
\end{tabular}

\section{Discussion}

The prevalence of depression among peoples living with HIV/AIDS in this study is $41.7 \%$. This finding is consistent with study done in Harar (45.8\%) [15], Tigray (43.9\%) [18] and China (40.9\%) [12]. However; it is lower than a study done in Fitche zonal hospital (76.7\%) [29] and Hawassa (48.6\%) [13]. On the other hand, it is higher than study done in Malawi (18.9\%) [11], Addis Ababa (35.5\%) [16], Aksum town (14.6\%) [14] and Cameroon (26.7\%) [10]. The difference might be due to variation in data collection tool, sample size and study participant's variation. The prevalence of depression among people living with HIV/AIDS in this study is much higher than the general population in Ethiopia (11\%) [6].

In this study, factors such as social support, perceived social stigma, opportunistic infection, adverse drug reaction and presence of other chronic diseases were significantly associated with depression. Study participant who have poor social support had more depression than those who have strong social support. This might be due to the fact that poor social support may leads to social isolation, which can be responsible for depression. This finding is consistent with study done in Addis Ababa [16], Hawassa [13] and China [12]. However; this finding is not consistent with study done in Cameroon [10], Harar [15], Aksum [14] and Malawi [11]. The finding of this study also showed that those participants who perceived social stigma had depression than who didn't perceived social stigma. This might be due to chronic nature of the disease and the patient may prefer being alone to avoid stigma which may further lead to the development of depression. This result is similar with studies done in Malawi [11], Addis Ababa [16], Hawassa [13] and China [12].

Respondents who developed adverse drug reaction had more depressive symptoms than who didn't develop any adverse drug reaction. This is due to the fact that ART side effects may disturb the normal functioning of HIV/ AIDS patients, so that patients may feel hopeless and develop depressive symptoms. This finding is consistent with study done in Aksum town [14] and China [12]. The finding of this study also showed that those who had history of opportunistic infection were more likely to develop depression. This might be due to the fact that opportunistic infection may lead to dissatisfaction with one's physical appearance, which might be a reason for the occurrence of depression. This finding is supported by study done in Addis Ababa [16] and Fitche zonal hospital [29].

In this study, respondents who had history of other chronic illness were more depressed than those who had no history of other chronic illness. This might be due to the occurrence of long-lasting stresses related to chronic 
Table 3 Multivariate logistic regression analysis of factors associated with depression among HIV/AIDS patients at Gimbi General hospital, West Ethiopia, $2018(\mathrm{n}=393)$

\begin{tabular}{|c|c|c|c|c|c|c|}
\hline \multirow[t]{2}{*}{ Variables } & \multicolumn{2}{|l|}{ Depression } & \multirow[t]{2}{*}{ COR (95\%) Cl } & \multirow{2}{*}{$\begin{array}{l}\text { Unadjusted P } \\
\text { value }\end{array}$} & \multirow[t]{2}{*}{ AOR (95\%) Cl } & \multirow{2}{*}{$\begin{array}{l}\text { Adjusted P } \\
\text { value }\end{array}$} \\
\hline & Yes (\%) & No (\%) & & & & \\
\hline \multicolumn{7}{|l|}{ Sex } \\
\hline Male & 72 (40.7\%) & 105 (59.3\%) & $0.924(0.61,1.38)$ & 0.70 & $1.2(0.8,1.86)$ & 0.35 \\
\hline Female & 92 (42.6\%) & 124 (57.4\%) & 1 & & 1 & \\
\hline \multicolumn{7}{|l|}{ Age (years) } \\
\hline $18-28$ & $58(40.8 \%)$ & 84 (59.2\%) & 1 & & 1 & \\
\hline $29-38$ & $57(40.7 \%)$ & $83(59.3 \%)$ & $0.99(0.61,1.59)$ & 0.98 & $0.82(0.28,2.36)$ & 0.72 \\
\hline $39-48$ & $22(39.3)$ & 34 (60.7\%) & $0.93(0.49,1.76)$ & 0.84 & $0.37(0.07,1.96)$ & 0.24 \\
\hline$>48$ & $27(49.1 \%)$ & $28(50.9 \%)$ & $1.39(0.74,2.61)$ & 0.29 & $0.82(0.22,3.07)$ & 0.77 \\
\hline \multicolumn{7}{|l|}{ Religion } \\
\hline Protestant & 75 (41.2\%) & 107 (58.8\%) & 1 & & 1 & \\
\hline Orthodox & $44(41.5 \%)$ & 62 (58.5\%) & $1.01(0.62,1.64)$ & 0.96 & $0.75(0.27,2.02)$ & 0.57 \\
\hline Muslim & $24(49.0 \%)$ & $25(51.0 \%)$ & $1.37(0.72,2.58)$ & 0.33 & $0.63(0.17,2.28)$ & 0.48 \\
\hline Catholic & $16(34.8 \%)$ & 30 (65.2\%) & $0.76(0.38,1.49)$ & 0.47 & $1.33(0.32,5.49)$ & 0.69 \\
\hline Others & $5(50.0 \%)$ & $5(50.0 \%)$ & $1.42(0.39,5.10)$ & 0.58 & $2.10(0.80,8.22)$ & 0.71 \\
\hline \multicolumn{7}{|l|}{ Marital status } \\
\hline Married & $51(38.6 \%)$ & $81(61.4 \%)$ & 1 & & 1 & \\
\hline Single & 73 (42.0\%) & 101 (58.0\%) & $1.14(0.72,1.82)$ & 0.55 & $0.55(0.20,1.53)$ & 0.25 \\
\hline Divorced & 22 (44.9\%) & 27 (55.1\%) & $1.29(0.66,2.51)$ & 0.44 & $0.95(0.23,3.89)$ & 0.94 \\
\hline Widowed & $18(47.4 \%)$ & $20(52.6 \%)$ & $1.42(0.69,2.95)$ & 0.33 & $0.83(0.13,5.26)$ & 0.84 \\
\hline \multicolumn{7}{|l|}{ Ethnicity } \\
\hline Oromo & 93 (39.7\%) & $141(60.3 \%)$ & 1 & & 1 & \\
\hline Amhara & 49 (46.2\%) & 57 (53.8\%) & $1.30(0.82,2.07)$ & 0.26 & $2.18(0.84,5.61)$ & 0.10 \\
\hline Tigre & $11(45.8 \%)$ & $13(54.2 \%)$ & $1.28(0.55,2.98)$ & 0.56 & $1.32(0.28,6.13)$ & 0.71 \\
\hline Gurage & $7(43.8 \%)$ & $9(56.2 \%)$ & $1.12(0.42,3.27)$ & 0.75 & $1.69(0.19,14.6)$ & 0.63 \\
\hline Others & $4(30.8 \%)$ & $9(69.2 \%)$ & $0.67(0.20,2.25)$ & 0.52 & $0.15(0.01,2.32)$ & 0.17 \\
\hline \multicolumn{7}{|l|}{ Educational status } \\
\hline No formal education & $13(43.3 \%)$ & $17(56.7 \%)$ & $1.20(0.50,2.88)$ & 0.67 & $1.08(0.15,7.50)$ & 0.93 \\
\hline Primary school (1-8) & $54(42.2 \%)$ & $74(57.8 \%)$ & $1.15(0.62,2.10)$ & 0.64 & $2.05(0.54,7.67)$ & 0.28 \\
\hline Secondary school (9-12) & $71(42.3 \%)$ & $97(57.7 \%)$ & $1.15(0.64,2.05)$ & 0.62 & $1.78(0.55,5.78)$ & 0.33 \\
\hline College and above & $26(38.8 \%)$ & $41(61.2 \%)$ & 1 & & 1 & \\
\hline \multicolumn{7}{|l|}{ Residence } \\
\hline Urban & $55(43.0 \%)$ & $73(57.7 \%)$ & $1.07(0.70,1.65)$ & 0.72 & $0.87(0.34,2.17)$ & 0.76 \\
\hline Rural & $109(41.1 \%)$ & $156(58.9 \%)$ & 1 & & 1 & \\
\hline \multicolumn{7}{|l|}{ Occupation } \\
\hline Government employee & $19(36.5 \%)$ & $33(63.5 \%)$ & 1 & & 1 & \\
\hline Private employee & $50(41.3 \%)$ & $71(58.7 \%)$ & $1.22(0.62,2.39)$ & 0.55 & $1.80(0.48,6.72)$ & 0.37 \\
\hline Farmer & $51(53.7 \%)$ & $44(46.3 \%)$ & $2.01(1.00,4.02)$ & $0.04^{*}$ & $1.50(0.38,5.91)$ & 0.55 \\
\hline Merchant & $43(39.4 \%)$ & $66(60.6 \%)$ & $1.13(0.57,2.24)$ & 0.72 & $2.06(0.54,7.77)$ & 0.28 \\
\hline Others & 1 (6.2\%) & 15 (93.8\%) & $0.11(0.01,0.94)$ & $0.04^{*}$ & $0.07(0.04,1.31)$ & 0.75 \\
\hline \multicolumn{7}{|l|}{ Income } \\
\hline$<500 \mathrm{~EB}$ & $51(47.2 \%)$ & $57(52.8 \%)$ & $0.62(0.28,1.36)$ & $0.24^{*}$ & $0.92(0.15,5.54)$ & 0.92 \\
\hline 500-1000 EB & 39 (39.8\%) & 59 (60.2\%) & $0.46(0.20,1.02)$ & $0.05^{*}$ & $0.49(0.07,3.09)$ & 0.44 \\
\hline $1001-1500$ EB & $29(37.2 \%)$ & 49 (62.8\%) & $0.41(0.18,0.94)$ & $0.03^{*}$ & $0.85(0.13,5.25)$ & 0.86 \\
\hline $1501-2000$ EB & $25(33.3 \%)$ & $50(66.7 \%)$ & $0.35(0.15,0.80)$ & $0.01^{*}$ & $0.63(0.10,3.80)$ & 0.62 \\
\hline$>2000 \mathrm{~EB}$ & $20(58.8 \%)$ & $14(41.2 \%)$ & 1 & & 1 & \\
\hline \multicolumn{7}{|l|}{ Living companion } \\
\hline Yes & 73 (27.7\%) & 191 (72.3\%) & 1 & & 1 & \\
\hline
\end{tabular}


Table 3 (continued)

\begin{tabular}{|c|c|c|c|c|c|c|}
\hline \multirow[t]{2}{*}{ Variables } & \multicolumn{2}{|l|}{ Depression } & \multirow[t]{2}{*}{ COR $(95 \%) \mathrm{Cl}$} & \multirow{2}{*}{$\begin{array}{l}\text { Unadjusted P } \\
\text { value }\end{array}$} & \multirow[t]{2}{*}{ AOR (95\%) Cl } & \multirow{2}{*}{$\begin{array}{l}\text { Adjusted } \\
\text { value }\end{array}$} \\
\hline & Yes (\%) & No (\%) & & & & \\
\hline No & $91(70.5 \%)$ & $38(29.5)$ & $6.26(3.93,9.97)$ & $0.01^{*}$ & $3.33(1.58,9.96)$ & 0.09 \\
\hline \multicolumn{7}{|l|}{ CD4 cell } \\
\hline$<200$ & $31(49.2 \%)$ & $32(50.8 \%)$ & $0.44(0.13,1.41)$ & $0.16^{*}$ & $0.49(0.04,5.70)$ & 0.57 \\
\hline $200-500$ & $70(38.7 \%)$ & $111(61.3 \%)$ & $0.28(0.096,0.86)$ & $0.02^{*}$ & $0.27(0.02,2.74)$ & 0.26 \\
\hline $501-800$ & $52(39.1 \%)$ & 81 (60.9\%) & $0.29(0.09,0.88)$ & $0.03^{*}$ & $0.31(0.03,3.14)$ & 0.32 \\
\hline$>800$ & $11(68.8 \%)$ & $5(31.2 \%$ & 1 & & 1 & \\
\hline \multicolumn{7}{|l|}{ HIV stage } \\
\hline Stage I & 45 (38.5\%) & $72(61.5 \%)$ & 1 & & 1 & \\
\hline Stage II & $49(32.0 \%)$ & $104(68.0 \%)$ & $0.75(0.45,1.24)$ & 0.27 & $0.74(0.31,1.76)$ & 0.50 \\
\hline Stage III & $33(45.8 \%)$ & $39(54.2 \%)$ & $1.35(0.74,2.45)$ & 0.31 & $2.15(0.74,6.23)$ & 0.15 \\
\hline Stage IV & $37(72.5 \%)$ & $14(27.5 \%)$ & $4.22(2.06,8.67)$ & $<0.001^{*}$ & $2.31(0.60,8.86)$ & 0.22 \\
\hline \multicolumn{7}{|c|}{ Family/social support } \\
\hline Poor & $45(65.2 \%)$ & $24(34.8 \%)$ & $2.79(1.56,4.99)$ & $<0.001^{*}$ & $9.97(3.57,27.86)$ & $0.000 * *$ \\
\hline Moderate & $48(32.7 \%)$ & $99(67.3 \%)$ & $0.72(0.45,1.14)$ & $0.16^{*}$ & $1.38(0.60,3.13)$ & 0.44 \\
\hline Strong & $71(40.1 \%)$ & 106 (59.9\%) & 1 & & 1 & \\
\hline \multicolumn{7}{|c|}{ Perceived stigma } \\
\hline Yes & $115(78.8 \%)$ & $31(21.2 \%)$ & $14.99(9.04,24.84)$ & $<0.001^{*}$ & $6.98(3.07,15.86)$ & $0.000^{* *}$ \\
\hline No & 49 (19.8\%) & 198 (80.2\%) & 1 & & 1 & \\
\hline \multicolumn{7}{|c|}{ Substance use } \\
\hline Yes & $128(81.5 \%)$ & $29(18.5 \%)$ & $24.52(14.33,41.95)$ & $0.04^{*}$ & $5.06(2.39,10.75)$ & 0.17 \\
\hline No & $36(15.3 \%)$ & $200(84.7 \%)$ & 1 & & 1 & \\
\hline \multicolumn{7}{|c|}{ Disease duration (years) } \\
\hline$<1$ & $20(37.0 \%)$ & $34(63.0 \%)$ & 1 & & 1 & \\
\hline $1-5$ & $53(38.7 \%)$ & $84(61.3 \%)$ & $1.07(0.56,2.05)$ & 0.83 & $0.64(0.14,2.78)$ & 0.55 \\
\hline $6-10$ & $74(50.0 \%)$ & $74(50.0 \%)$ & $1.70(0.89,3.22)$ & $0.10^{*}$ & $1.37(0.32,5.77)$ & 0.66 \\
\hline$>10$ & $17(31.5 \%)$ & $37(68.5 \%)$ & $0.78(0.35,1.73)$ & 0.54 & $0.58(0.10,3.24)$ & 0.54 \\
\hline \multicolumn{7}{|c|}{ Treatment duration (years) } \\
\hline$<1$ & $27(37.5 \%)$ & $45(62.5 \%)$ & 1 & & 1 & \\
\hline $1-5$ & $65(39.6 \%)$ & $99(60.4 \%)$ & $1.09(0.61,1.93)$ & 0.75 & $2.35(0.77,7.14)$ & 0.13 \\
\hline $6-10$ & $68(48.6 \%)$ & $72(51.4 \%)$ & $1.57(0.88,2.81)$ & $0.12^{*}$ & $4.0(1.28,12.51)$ & 0.17 \\
\hline$>10$ & $4(23.5 \%)$ & $13(76.5 \%)$ & $0.51(0.15,1.73)$ & 0.28 & $3.21(0.40,25.69)$ & 0.20 \\
\hline \multicolumn{7}{|c|}{ Adherence to medication } \\
\hline Yes & $59(24.1 \%)$ & $186(75.9 \%)$ & 1 & & 1 & \\
\hline No & 105 (70.9\%) & $43(29.1 \%)$ & $7.69(4.85,12.19)$ & $0.003^{*}$ & $4.92(1.84,13.11)$ & 0.061 \\
\hline \multicolumn{7}{|c|}{ Adverse drug reaction } \\
\hline Yes & $103(82.4 \%)$ & $22(17.6 \%)$ & $15.88(9.24,27.30)$ & $<0.001^{*}$ & $3.73(1.58,8.81)$ & $0.003^{* *}$ \\
\hline No & $61(22.8 \%)$ & $207(77.2 \%)$ & 1 & & 1 & \\
\hline \multicolumn{7}{|c|}{ History of other chronic illness } \\
\hline Yes & $20(66.7 \%)$ & $10(33.3 \%)$ & $3.04(1.38,6.68)$ & $0.006^{*}$ & $6.14(1.66,22.68)$ & $0.006^{* *}$ \\
\hline No & $144(39.7 \%)$ & $219(60.3 \%)$ & 1 & & 1 & \\
\hline \multicolumn{7}{|c|}{ Opportunistic infection } \\
\hline Yes & $89(72.4 \%)$ & $34(27.6 \%)$ & $6.80(4.22,10.96)$ & $<0.001^{*}$ & $9.38(4.21,20.89)$ & $0.000^{* *}$ \\
\hline No & 75 (27.8\%) & 195 (72.2\%) & 1 & & 1 & \\
\hline
\end{tabular}

**Significant at P-value $<0.05$

diseases which may further leads to depressive symptoms. This finding is consistent with study conducted at Debrebirhan Referral Hospital [30]. Substance use, living companion, medication adherence and being female had no statistically significant association with depression in this study. 


\section{Conclusion}

The level of depression among HIV/AIDS patient in this study was high. Depression among people living with HIV/AIDS is underdiagnosed and further largescale research is needed. The policy makers should integrate mental health programs into routine HIV care for early identification and treatment of depression. The health workers should focus on patients both clinical and social aspect taking presence of social support, social stigma, adverse drug reaction, presence of opportunistic infection and other chronic diseases into great consideration.

\section{Limitation of the study}

Causality cannot be confirmed since the research design is cross-sectional. This study was conducted at single hospital. Thus, the finding cannot be generalized to Ethiopian people and caution is warranted in generalizing the findings to other areas.

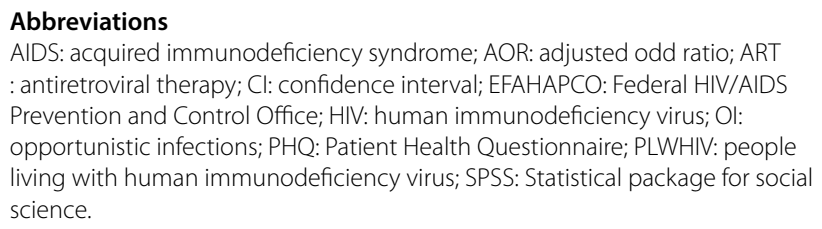

Abbreviations

AIDS: acquired immunodeficiency syndrome; AOR: adjusted odd ratio; ART : antiretroviral therapy; Cl: confidence interval; EFAHAPCO: Federal HIV/AIDS Prevention and Control Office; HIV: human immunodeficiency virus; OI: opportunistic infections; PHQ: Patient Health Questionnaire; PLWHIV: people living with human immunodeficiency virus; SPSS: Statistical package for social science.

\section{Acknowledgements}

I would like to acknowledge Gimbi General hospital medical director and staffs for their cooperation. I am also grateful to the study participants who voluntarily agreed to be interviewed and participated in the study.

\section{Authors' contributions}

Overall activities of this study (conceptualization of the study, designing, result writing, analyzing the finding and writing the manuscript) were carried out by MA. The author read and approved the final manuscript.

\section{Funding}

No funding was received for this research work.

\section{Availability of data and materials}

The data used during this study are available on request.

\section{Ethics approval and consent to participate}

The study was reviewed and approved by the Institutional Review Boards of Wollega University Ethical review board. The purpose of the study was explained to medical director and staffs of the hospital and permission was obtained. All participants of the study were provided written consent, clearly stating the objectives of the study and their right to refuse. Filled out questionnaires were carefully handled and all access to results was kept strictly within the author.

\section{Consent for publication}

Not applicable.

\section{Competing interests}

The author declares no competing interests.

Received: 31 May 2019 Accepted: 10 August 2019 Published online: 20 August 2019

\section{References}

1. Saxena S, Funk M, Chisholm D. WHO's Mental Health Action Plan 2013-2020: what can psychiatrists do to facilitate its implementation? World Psychiatry. 2014;13:107-9.

2. World Federation for Mental Health. Depression: a global crises book. 2012. p. 6. https://www.who.int/mental_health/management/depression /wfmh_paper_depression_wmhd_2012.pdf.

3. World Health Organization. Depression fact sheet on mental health. https ://www.who.int/campaigns/world-health-day/2017/fact-sheets/en/. Updated Feb 2017.

4. WHO. Depression: a global public health concern. 2012. https://www. who.int/mental_health/management/depression/who_paper_depre ssion_wfmh_2012.pdf.

5. WHO. Mental health. 2012. https://www.who.int/mental_health/preve ntion/suicide/suicideprevent/en/2012.

6. Tesera B. Prevalence of depression and potential risk factors in Ethiopia. Ethiop J Health Sci. 2014;24(2):161-9.

7. Prince M, Patel V, Saxena S, Maj M, Maselko J, et al. No health without mental health. Lancet. 2007;370:859-77.

8. WHO. Mental health and development: targeting people with mental health condition as a vulnerable group. Geneva: World Health Organization; 2010

9. Silveira MP, Guttier MC, Pinheiro CA, Pereira TV, Cruzeiro AL, Moreira LB. Depressive symptoms in HIV-infected patients treated with highly active antiretroviral therapy. Braz J Psychiatry. 2012;34(2):162-7.

10. Ngum PA, de Fon PN, Ngu RC, Verla VS, Luma HN. Depression among HIV/aids patients on highly active antiretroviral therapy in the southwest regional hospitals of Cameroon: a cross-sectional study. Neurol Ther. 2017;6:103-14. https://doi.org/10.1007/s40120-017-0065-9.

11. Kim, et al. Factors associated with depression among adolescents living with HIV in Malawi. BMC Psychiatry. 2015;15:264. https://doi.org/10.1186/ s12888-015-0649-9.

12. Liu $\mathrm{H}$, et al. Identifying factors associated with depression among men living with HIV/AIDS and undergoing antiretroviral therapy: a crosssectional study in Heilongjiang, China. Health Qual Life Outcomes. 2018;16:190. https://doi.org/10.1186/s12955-018-1020-x.

13. Duko, et al. Prevalence and associated factors of depression among patients with HIV/AIDS in Hawassa, Ethiopia, cross-sectional study. Ann Gen Psychiatry. 2018;17:45.

14. Berhe et al. Depression among adult HIV/AIDS patients attending ART clinics at Aksum Town, Aksum, Ethiopia: a cross-sectional study. Depression Res Treat. 2019, Article ID 3250431, 8 pages.

15. Mohammed M, Mengistie B, Dessie Y, Godana W. Prevalence of depression and associated factors among HIV patients seeking treatments in ART Clinics at Harar Town, Eastern Ethiopia. J AIDS Clin Res. 2015;6:474. https://doi.org/10.4172/2155-6113.1000474.

16. Shumet et al. Prevalence of depressive symptoms and associated factors among HIV-positive youth attending ART follow-up in Addis Ababa, Ethiopia. AIDS Res Treat. 2019, Article ID 4610458, 7 pages.

17. Rabkin JG. HIV and depression: 2008 review and update. Curr HIV/AIDS Rep. 2008;5:163-71.

18. Tucker JS, Burnam MA, Sherbourne CD, Kung FY, Gifford AL. Substance use and mental health correlates of nonadherence to antiretroviral medications in a sample of patients with human immunodeficiency virus infection. Am J Med. 2003;114:573-80.

19. Berger-Greenstein JA, Cuevas CA, Brady SM, Trezza G, Richardson MA, et al. Major depression in patients with HIV/AIDS and substance abuse. AIDS Patient Care STDS. 2007;21:942-55.

20. Mathers CD, Loncar D. Projections of global mortality and burden of disease from 2002 to 2030. PLoS Med. 2006;3:e442.

21. WHO. HIV/AIDS and mental health: report by the secretariat. Geneva: World Health Organization; 2008.

22. Abas M, Ali G-C, Nakimuli-Mpungu E, Chibanda D. Depression in people living with HIV in sub-Saharan Africa: time to act. Trop Med Int Health. 2014;19:1392-6.

23. Uthman OA, Magidson JF, Safren SA, Nachega JB. Depression and adherence to antiretroviral therapy in low-, middle- and high-income countries: a systematic review and meta-analysis. Curr HIV/AIDS Rep. 2014;11:291-307. 
24. Chaudhury S, Bakhla AK, Saini R. Prevalence, impact, and management of depression and anxiety in patients with HIV: a review. Neurobehav HIV Med. 2016;7:15-30

25. Gelaye B, Williams MA, Lemma S, et al. Validity of the patient health questionnaire-9 for depression screening and diagnosis in East Africa. Psychiatry Res. 2013;210(2):653-61.

26. Dalgard OS, Dowrick C, Lehtinen V, Vazquez-Barquero JL, Casey P, Wilkinson $\mathrm{G}$, et al. Negative life events, social support and gender difference in depression. Soc Psychiatry Psychiatr Epidemiol. 2006;41(6):444-51.

27. Van Rie A, Sengupta S, Pungrassami P, Balthip Q, Choonuan S, Kasetjaroen $Y$, et al. Measuring stigma associated with tuberculosis and HIV/AIDS in southern Thailand: exploratory and confirmatory factor analyses of two new scales. Trop Med Int Health. 2008;13(1):21-30.

28. Franke MF, Muñoz M, Finnegan K, Zeladita J, Sebastian JL, Bayona JN, Shin SS. Validation and abbreviation of an HIV stigma scale in an adult spanish speaking population in urban Peru. AIDS Behav. 2010;14:189-99.
29. Yeneabat T, et al. Factors associated with depressive symptoms in people living with HIV attending antiretroviral clinic at Fitche Zonal hospital, central Ethiopia. Neuropsychiatr Dis Treat. 2017;13:2125-31.

30. Asmare, et al. Prevalence of depression and associated factors among HIV/AIDS patients attending ART clinic at Debrebirhan Referral Hospital, North Showa, Ethiopia. Clin Psychiatry. 2015;1(1):3.

\section{Publisher's Note}

Springer Nature remains neutral with regard to jurisdictional claims in published maps and institutional affiliations.
Ready to submit your research? Choose BMC and benefit from:

- fast, convenient online submission

- thorough peer review by experienced researchers in your field

- rapid publication on acceptance

- support for research data, including large and complex data types

- gold Open Access which fosters wider collaboration and increased citations

- maximum visibility for your research: over 100M website views per year

At BMC, research is always in progress.

Learn more biomedcentral.com/submissions 